\title{
The need for faculty training programs in effective feedback provision
}

\author{
This article was published in the following Dove Press journal: \\ Advances in Medical Education and Practice \\ 21 August 2014 \\ Number of times this article has been viewed
}

\author{
Abdullah Al Wahbi ${ }^{1,2}$ \\ 'King Saud University for Health \\ Sciences, ${ }^{2}$ King Abdulaziz Medical \\ City, Division of Vascular Surgery, \\ Department of Surgery, Riyadh, \\ Saudi Arabia
}

Correspondence: Abdullah Al Wahbi Division of Vascular Surgery, Department of Surgery, King Abdulaziz Medical City, Mail code 1446, PO Box 22490,

Riyadh II426, Saudi Arabia Email amw8855@gmail.com; wahbia@ ngha.med.sa

\begin{abstract}
An important aspect of professional teaching practice is a practitioner's ability to critically evaluate the performances of subordinates for whom he or she is responsible. This is a common practice within social sciences as well as for professionals from applied specialties. The literature on professional clinical expertise identifies reflective practice as perfect when they are thoroughly accepted by practitioners. In health-related professions, critical reflection in the form of feedback that serves as the bridge between theory and practice is endorsed. The aims and objectives of this study were directed toward the application of a mixed methodology approach in order to evaluate the requirements for a feedback training program and to detect the present feedback provision skills of clinical mentors in practice. The quantitative analysis measured the effectiveness of clinical teachers' feedback in order to understand whether their understanding of and skills for giving feedback to promote students were adequate. On the other hand, the qualitative methods explored self-perceptions of feedback skills and efficacy in enabling students to improve their clinical practice. Effective feedback from faculty and the learner provides a useful and meaningful experience for absorbing knowledge and critical thinking into clinical practice. Nonadherence and limited expertise of mentors in giving feedback are the main themes of this study, and were evaluated and acknowledged through systematic analysis.
\end{abstract}

Keywords: clinical mentors, feedback mechanism, feedback proficiency

\section{Introduction}

Feedback from clinical mentors to students is essential for introducing successful professional practice and needs to be promoted throughout the professional curriculum. ${ }^{1}$ The ability of teachers to provide positive and constructive feedback to students relies on teachers themselves acknowledging factors that can facilitate the execution of their duties in a better way. ${ }^{2}$ The traditional written feedback system was flawed as it did not tend to motivate students adequately to do better nor was it capable of identifying the weaknesses of students. ${ }^{3}$ This study evaluates teachers' self-awareness about and proficiency in giving feedback to students to improve their clinical skills. This report intends to explore the need for an effective and successful training program of feedback provision of clinicians. Due to the critical and complex nature of medical practices, health care instructors face barriers in implementing verbal feedback in clinical settings. ${ }^{4}$ This study questions the levels of efficiency of the feedback mechanism of teachers working in various clinical settings in King Abdulaziz Medical City in Riyadh, Saudi Arabia. 


\section{Literature review}

This study aims to assess and evaluate the hallmarks of clinical education in order to promote feedback skills and to acknowledge the perceived and real feedback skills of clinical teachers. The value of feedback in clinical education is not only in improving care and services for patients, but that it is also critical for overall clinical processes and student learning. If feedback techniques are not properly implemented, the learner competency can never be achieved and clinical practice errors cannot be rectified. ${ }^{5}$

\section{Geis' instructional feedback model}

According to Geis' instructional method, evaluation and scrutiny in a clinical setting are considered components of the change process and have a potential effect on the utilization of feedback by the learner. In this model, the recipient establishes the positive or negative use of the feedback through contrasting variables, which include the implication of improvement scenarios experienced by a recipient previously and a recipient's ability to identify and correct issues in their performance.

\section{The role of the clinical educator}

Feedback comprises the core of medical teaching, but few clinicians are aware of its effectiveness and of the principles to be followed in ways versus patterns that are productive and essential for elevating the learning capabilities of the students. Further, there are different teaching feedback mechanisms for every teaching speciality, ${ }^{6}$ and the role of the teacher in clinical practice requires the adoption of dual roles - those of both a collaborator and of a scholar. ${ }^{7}$ Teachers need to possess four fundamental abilities in order to depict better prospects in students, and these are facilitation of experimental learning; facilitation of progress and socialization; the implications and evaluation of techniques; and active contributions in curriculum designing. ${ }^{7}$ The role of collaborator involves learner competency as a leader and as a medium for bringing about change in the system, as well as involvement in attaining scholarships, and these are deemed to be basic requirements of a clinical instructor. The scholarly role concerns the prominent integrated concepts of competence such as achieving regular and sustained improvement in learning. When referring to education practice, the core of a constructive framework that could be implemented in a context of individual progress requires subsequent evaluation of the students in clinical practice. ${ }^{?}$

\section{Feedback mechanisms}

The higher cognitive centers in adults are specialized in adopting the perception of learning and reasoning, and then interpreting them into intellect by accumulation of various experiences. Panasuk and Lebaron identified that adult learners have strong abilities to link learned theoretical knowledge with experimental learning experiences, ${ }^{8}$ while Howley and Martindale illustrated that medical learners are self-motivated to learn and possess desire to be recognized in professional settings; in order to achieve these outcomes, they seek feedback and reinforcement about their performances and future goals. ${ }^{9}$

By implementing the critical thinking strategies incorporated into learning according to the students' needs and requirements, the instructor can meet the challenges of the clinical environment. ${ }^{8}$ The feedback process needs to focus on issues concerning students' life experiences, learning styles, and outcomes; course planning and expectations; and students' recognition of learning and education. The utilization of different feedback instruments addresses issues related to appropriateness of course objectives, methodology followed, techniques of execution, and organization of data and results. ${ }^{8}$

\section{Categories of feedback techniques}

Feedback can only be successful in attaining related goals when the relationship between the instructors and the pupils is strong and cohesive. ${ }^{10}$ The dynamics of the roles that lie between these two entities vary from one faculty to another. Dialogue is a type of feedback that is communicative, cooperative, and conservative in origin. The real meaning of dialogue is the sharing of views, ideas, and insights of both parties to form an inference that is productive in nature. ${ }^{11}$

According to Anderson, dialogues enable the transformation of beliefs and relationships between instructors and learners necessary for critical thinking and complex learning. ${ }^{12}$ Cooperative discussions require social independence of the learners in order that they can express their ideas through structured and planned interactive sessions. ${ }^{10}$ Teacher-tostudent feedback assists students in clarifying their goals and identifying their mistakes as well as establishing their sense of direction. ${ }^{10}$ This form of feedback transmits knowledge to learners and makes the understanding process of what has been taught easier for them to appreciate.

Hasley and Arnold described summative feedback as positive and corrective statements with the aim of improving the level of performance of the learners. ${ }^{11}$ Simulated patient feedback in clinical teaching and assessment is vital for the faculty to gather a better understanding of students' skills in data collection, solution finding, and managing clinical information. ${ }^{9}$ Feedback between teachers is essential in a feedback training program, and that includes the identification of aspects of 
student mentorship and recognizing the shortcomings in the teaching process. It also emphasizes the potential of the students to achieve success by identifying what students could do to make a difference. ${ }^{13}$

\section{The requirements for feedback}

According to Bruner, there are four modes of learning: 1) learning by seeing; 2) learning by listening; 3) learning through experimental or constructive methods; and 4) learning by observing those with greater knowledge. ${ }^{14}$ Student dissatisfaction is often based on receiving insufficient guidance in feedback. The consistent provision of feedback in medicine is not easy to carry out for two reasons: first, trainees may be selected based on inaccurate information or insufficient skills, and second is the unease felt by clinical instructors in delivering feedback. ${ }^{9}$

The effectiveness of workshops for written feedback lies in the feedback correlating the grades of students with concrete reasons and providing suggestions for improvement. ${ }^{15}$ Current study suggests that the feedback mechanisms used by our participants are conventional, generic in nature, and often contain just criticism from the instructors. Feedback needs to be simple but corrective in order to address the issues that hinder students' improvement in clinical performance. ${ }^{9}$

In the field of clinical practice, learners need to acquire skills coupled with intellectual, cognitive, and psychosomatic domains. In order to improve trainees' skills, doctors' feedback on observed practices and evaluation are essential. Feedback provision is a vital part of the learning process, as learners receive instruction about how well they are doing and how they can improve.

\section{Methodology}

Creswell suggests that a mixed methodological approach addresses the issues affecting the growth of professional development. The qualitative and quantitative research methods, when applied together and combined with statistical techniques, lead to better discussion of result findings. ${ }^{16}$ A questionnaire was constructed to measure effectiveness of clinical teachers in providing feedback to students by incorporating the descriptive statistics and correlation techniques and by applying Statistical Package for Social Science (version 21: IBM Corporation, Armonk, NY, USA) methodology for evaluation.

Qualitative design enables assessment of the observed behaviors, which can then be implemented to enhance the proficiency of instructors and how faculty members perform. ${ }^{16}$ Complementary issues, such as the barriers that teachers face in giving feedback, were addressed through qualitative data analysis and information was gathered through open-ended survey questions. The quantitative design assisted in the identification of the weaknesses of this study and enabled the researcher to measure the feedback proficiency scores of the teachers. The quantitative data collection involved the close-ended items in the survey.

The questionnaire was sent to 60 respondents who were acting as clinical instructors in internal medicine, intensive care, family physicians, pediatrics, and surgery specialties in King Abdulaziz Medical City in Saudi Arabia. These participants were responsible for providing clinical feedback to student interns at the hospital governed by the University Faculty Development Program. The respondents were recruited through emails and were asked to fill out a questionnaire to assess their response to student performance. These students were medical students who were exposed to different diagnoses and who were expected to perform various clinical duties during their internship.

Respondents were clinical instructors and were studied with the aim of canvassing their professional effectiveness at the feedback mechanism. The questionnaire consisted of introductory appendices regarding clinical experience, teaching backgrounds, and the type of students they mostly teach. The overall criteria for measuring the grade of the participant was extracted from the scoring in self-assessment and case scenario sections, with 10 as the maximum score and $50 \%$ as the passing score for this study.

The validity of the study process was strengthened by the pilot testing approach by the researcher. Only 20 faculty members responded, which elevates the reliability of the study to a small extent, but limits generalization to other populations. A research ethics committee approved the research and consent was obtained from all respondents with strict confidentiality and anonymity in context.

\section{Results}

According to the data collected, nearly one-half of the respondents failed the cumulative multiple choice questions (MCQ) and case scenario and only $47.4 \%$ passed the survey, with an overall response rate of $33 \%$. This finding signifies that there is a need for half of the faculty members to participate in feedback training programs in order to enhance their proficiency and effectiveness in giving feedback. Around $40 \%$ of the faculty members perceived their feedback proficiency to be better than their actual proficiency status. Only $10 \%$ of the participants truly acknowledged their lack of competency in feedback provision. These results verify the hypothesis that there was a lack of knowledge and application of effective feedback principles 
Percentage of faculty members with proficiency scores according to specialty

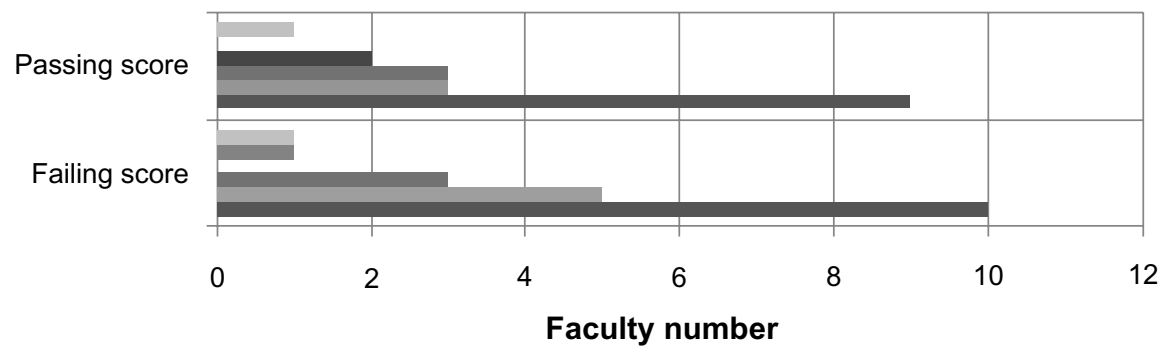

\begin{tabular}{|c|c|c|}
\hline & Failing score & Passing score \\
\hline Internal medicine & 1 & 1 \\
\hline Intensivist & 1 & 0 \\
\hline Family medicine & 0 & 2 \\
\hline Pediatric & 3 & 3 \\
\hline Surgery & 5 & 3 \\
\hline Total & 10 & 9 \\
\hline
\end{tabular}

Figure I Percentage of faculty members with passing and failing proficiency scores according to specialty.

among the clinical teachers of the program, even for those with a degree in medical education (Figure 1).

The impact of educational background on the proficiency of faculty members revealed that nine respondents who failed the MCQ test for feedback proficiency possessed degrees in medical education in clinical feedback provision. On the other hand, out of ten respondents who passed the MCQ test, eight of them did not have a degree in medical education in clinical instruction. However, $35 \%$ considered their effectiveness to be of a passing level and $10 \%$ incorrectly predicted that they would not pass the test (Figure 2).

Respondents gave the following as barriers they face in giving feedback to clinical students: insufficient office time; busy clinical schedule; no office hours; increased number of

Percentage of faculty members with a degree in medical education who have passed the MCQs

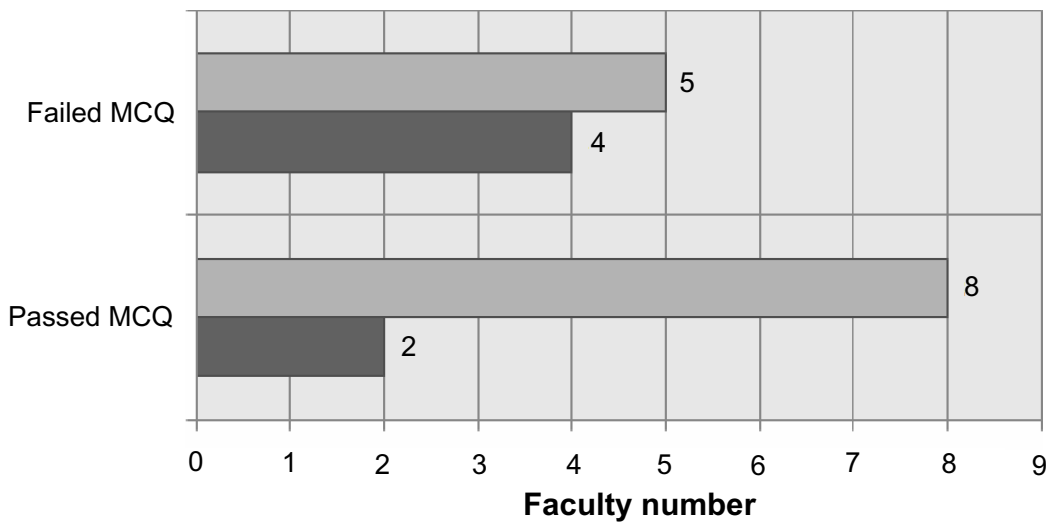

\begin{tabular}{l|c|c|}
\cline { 2 - 3 } & Passed MCQ & Failed MCQ \\
\hline$\square$ Without a degree in medical education & 8 & 5 \\
\hline$\square$ With a degree in medical education & 2 & 4 \\
\hline
\end{tabular}

Without a degree in medical education

With a degree in medical education

Figure 2 Percentage of faculty members with and without a degree in medical education who passed and failed multiple choice questions (MCQs). 
students; rapid resident turnover; lack of secretarial support; too many assignment tasks; and the students' training period being too short. This emphasizes the need for clinical teachers to have formal teaching programs to increase their feedback skills that take into account the barriers faced and suggests that regulations are required in clinical practice to cater for barriers to effective feedback provision.

\section{Discussion}

The implications of a constructive feedback mechanism are essentially based on learner proficiency of the clinical mentors. According to the findings, there were six faculty members who reported that they possess a degree in medical education in clinical instruction, while 13 others reported the inverse. Most of the faculty members without a degree in medical education had inaccurate perceptions about their competency in feedback provision as they view their skills to be compatible.

About $50 \%$ of the respondents with a degree had an exact match between their perceived and actual proficiency levels and believed that they had acceptable feedback skills. However, the availability of a degree in medical education did not appear to be a deciding factor between better or worse outcomes, as the respondents who did not have an academic qualification in clinical instruction passed the proficiency test comfortably. Further, just under one-half of the faculty members had accurate perceptions about their proficiency skills.

As per the findings indicated, the department of surgery had the most failing scores for feedback proficiency. All faculty from the internal medicine department had passing scores. Half of the pediatric department faculty had passing scores in the proficiency test but, due to uneven distribution of faculty members in various departments, the validity of these findings could not be established.

Feedback can be given in various formats; however, the inability of mentors to provide on-time and specific verbal feedback may prevent students from achieving their full potential. In general, participants who claimed to possess a qualification in clinical instruction scored lower than their expected scores. The reason for this may be due to their lack of regular practice in the relevant field or because their studies had little focus on feedback provision.

\section{Faculty response to feedback restraints}

The faculty members identified a busy schedule, as well as lack of office time, high student capacity, and lack of assistance in carrying out their jobs, as the basic restriction to their feedback performance. The increment in the advocacy for the students and ability of the teachers to acknowledge the importance of the feedback in medical practice and education were regarded as the implications for better feedback mechanism. However, the expertise of mentors greatly affects the learning capacity and outcomes of medical students.

When the instructors do not know their own proficiency levels, then it is difficult for them to provide effective feedback and correct responses for student benefit. The clinician must be able to provide feedback that is powerful and enables the pupils to progress. ${ }^{17}$ The absence of role models for proficient feedback provision has caused discrepancies in clinical practice. The results observed in this study indicate that there is a need for a faculty development plan or program that can incorporate feedback-developing skills and dialogues for constructive provision of knowledge for the clinical students.

\section{Limitations of the study}

The limitations of this study are the unavailability of the feedback report from directors of the student's program and communication skills, as these were indicated as barriers for competence. Further, during the study, it was suggested that trainees' emotional and ethnographic orientation also make it difficult for the mentor to state criticism in a way accepted by students. This study has only evaluated the efficiency levels of clinical mentors and has not investigated the satisfaction level of students receiving the feedback from them.

\section{Conclusion}

This study design has incorporated the collected data regarding the self-assessment criteria for measuring proficiency of a feedback mechanism by which clinical teachers could be more equipped to cater to the needs of medical students and enhance the learning procedures for the students. The results of the study indicate that the clinicians who responded had limited skills in feedback provision and most of them possessed inaccurate perceptions of their feedback proficiency. The acquisition of a professional degree has no implications on the clinicians' proficiency in providing feedback. The findings also emphasize that there is an immediate need for feedback training programs in order to promote better learning among clinical students. The conclusion extracted from this study is that neither educational background nor specialization acquirement has any impact on an individual's proficiency for giving feedback. Future research needs to focus on the factors that showed a link between feedback-giving proficiency and an 
individual's educational background. Feedback workshops and formal training programs can serve as the grounds for instilling clinical feedback skills in professionals, and also the feedback providence needs to be made compulsory in an overall institutional culture and dynamics.

\section{Disclosure}

The author reports no conflicts of interest in this work.

\section{References}

1. Bain JD, Ballantyne R, Packer J, Mills C. Using journal writing to enhance student teachers' reflectivity during field experience placements. Teachers and Teaching: Theory and Practice. 1999;5:51-73.

2. Baird MA. The idea of a reflective practicum: overcoming the dichotomy between academia and the practice setting. Radiography. 1996;2: 119-138.

3. Clouder L. Reflective practice in physiotherapy education: a critical conversation. Studies in Higher Education. 2000;25:211-223.

4. Mann K, Gordon J, MacLeod A. Reflection and reflective practice in health professions education: a systematic review. Adv Health Sci Educ Theory Pract. 2009;14(4):595-621.

5. McGaghie WC, Issenberg SB, Cohen ER, Barsuk JH, Wayne DB. Does simulation-based medical education with deliberate practice yield better results than traditional clinical education? A meta-analytic comparative review of the evidence. Acad Med. 2011;86(6):706-711.

6. Branch WT Jr, Paranjape A. Feedback and reflection: teaching methods for clinical settings. Acad Med. 2002;77(12):1185-1188.
7. Ficksman M, Adelizzi JU, editors. The Clinical Practice of Educational Therapy: A Teaching Model. Routledge; 2013.

8. Panasuk RM, Lebaron J. Student feedback: a tool for improving instruction in graduate education. Education. 1999;120(2):356.

9. Howley LD, Martindale J. The efficacy of standardized patient feedback in clinical teaching: a mixed methods analysis. Medical Education Online. 2004;9(18):1-10.

10. Carnell E. Dialogue, discussion, and feedback - views of secondary school students on how others help their learning. In: Askew S, editor. Feedback for Learning. London: RoutledgeFalmer; 2000:46-62.

11. Hasley PB, Arnold RM. Summative evaluation on the hospital wards. What do faculty say to learners? Adv Health Sci Educ Theory Pract. 2009; 14(3):431-439.

12. Anderson H. Collaborative learning communities. In: McNamee S, Gergen K, editors. Relational Responsibilities: Resources for Sustainable Dialogue. Thousand Oaks, CA: Sage Publications; 1999: 65-70.

13. Watkins C. Feedback between teachers. In: Askew S, editor. Feedback for Learning. London: Routledge Falmer; 2000:65-80.

14. Bruner JS. The Culture of Education. Cambridge, MA: Harvard University Press; 1996.

15. Salerno SM, Jackson JL, O’Malley PG. Interactive faculty development seminars improve the quality of written feedback in ambulatory teaching. Innovations in Education and Clinical Practice. 2003;18: 831-834.

16. Creswell JW. Research Design: Qualitative, Quantitative, and Mixed Methods Approaches. Thousand Oaks, CA: Sage Publications; 2003.

17. Palomba CA, Banta TW, editors. Assessing Student Competence in Accredited Disciplines: Pioneering Approaches to Assessment in Higher Education. Sterling, VA: Stylus Publishing; 2001.
Advances in Medical Education and Practice

\section{Publish your work in this journal}

Advances in Medical Education and Practice is an international, peerreviewed, open access journal that aims to present and publish research on Medical Education covering medical, dental, nursing and allied health care professional education. The journal covers undergraduate education, postgraduate training and continuing medical education

\section{Dovepress}

including emerging trends and innovative models linking education, research, and health care services. The manuscript management system is completely online and includes a very quick and fair peer-review system. Visit http://www.dovepress.com/testimonials.php to read real quotes from published authors. 